\title{
Ventricular Fibrillation in a 7-day-old Canine Model of Myocardial Infarction Role of Inhomogeneous Sympathetic Innervation
}

\author{
Satoshi Ogawa, MD
}

\begin{abstract}
Anstract
Ventricular fibrillation has been shown to be a leading cause of sudden death in post-myocardial infarction patients. Among various factors that modulate myocardial electrophysiologic properties, the autonomic tone is an important factor that can alter the ventricular fibrillation threshold. The increased dispersion of refractoriness and the propensity to ventricular tachyarrhythmias observed during sympathetic stimulation have been attributed to a non-uniform distribution of sympathetic transmitters throughout the myocardium. Heterogeneous sympathetic denervation contributes to a prolongation and dispersion of the effective refractory period in the surviving epicardium overlying the infarct. In this study, we evaluated local sympathetic innervation by measuring the changes in effective refractory periods (ERP) during ansae subclaviae stimulation (ASS) and norepinephrine infusion (NE). Sympathetic innervation was judged normal (N) when ERP shortening during ASS was $>5 \%$, denervated (DN) when ERP changes were $0-5 \%$, and supersensitive (DNS) when ERP shortening during NE was increased $(>12 \%)$ at denervated sites. ERP prolongation of $>5 \%$ during $\mathrm{ASS} / \mathrm{NE}$ was considered to represent increased $\alpha$-receptor sensitivity $(\alpha)$. During ERP determination, ventricular fibrillation was induced at 31 sites in 10 dogs by $\mathrm{S} 2$ at $\mathrm{S} 1-\mathrm{S} 2$ intervals $2-6$ msec longer than the ERP during ASS or NE.

Results: The ERP was determined at 283 sites in 10 dogs with inducible VF (group A) and 285 sites in 8 dogs without $\mathrm{VF}$ (group B). The incidence of DN and $\alpha$ in group $A$ was higher than that in group $B(43.5 \%$ vs $22.1 \%$, and $9.5 \%$ vs $1.8 \%$. Approximately $70 \%$ of the sites at which VF was induced in group A were normally innervated with ERP shortening of $>5 \%$ during ASS, while $83 \%$ of these sites were surrounded by either DN- or $\alpha$ areas, or both, at which ERP did not shorten or even prolonged during ASS/NE.

Of 31 sites, 12 sites were histologically normal and 13 sites were located at the periphery of the transmural infarct. The infarct size $(\%$ of the total left ventricular weight) was equal between groups $\mathrm{A}$ and $\mathrm{B}(24.1 \%$ vs $21.1 \%)$.

Conclusions: Heterogeneous sympathetic innervation contributed to an ERP dispersion in the surviving epicardium overlying the infarct. A singlc premature stimulus delivered from the peripheral region where areas with shorter ERP were surrounded by denervated areas or areas with increased $\alpha$ activity, could readily induce VF.
\end{abstract}

\footnotetext{
From the Cardiopulmonary Division, Department of Medicine, Keio University School of Medicine, Tokyo, Japan.
} 